\title{
A Linear Iteration Image Restoration Method Based on Homology Continuity
}

\author{
Yonghua Zhu, Shunyi Mao, Pin Wu, Honghao Gao and Zhiguo Wu \\ School of Computer Engineering and Science Shanghai University, China \\ Institute of Materials Genome, Shanghai University, Shanghai, China \\ Computing Center, Shanghai University, China \\ zyh@shu.edu.cn
}

\begin{abstract}
A novel image restoration method based on homology continuity is proposed in this paper. We view images as a collection of gray scale points, taking advantage of the homology continuity principle to combine each point and its fuzzy point derived by drop mass function to constitute the path direction and obtain distinct restore points. The gather of all the restore points is the sharply focused image of the original image. At last, we found every picture by iteration method is clearer than the last by experiment with the method proposed in the paper. The result verifies its feasibility.
\end{abstract}

Keywords: homology continuity, image restoration method, linear iteration, homologous image space, equivalent conditions of the image

\section{Introduction}

With the development of digital media technology, people have increasingly higher demands of digital image clarity. In the process of image acquisition, transmission and storage, image quality may deteriorate due to various reasons such as blurring, distortion, noise, etc. Such background puts forward urgent requirements for us to make image processing, in order to recover the possible original appearance, which is of significant importance in building the current digital database.

Image distortion refers to the difference between digital images and real shooting environment, which is caused by the internal image processors of sensors, optical systems and digital cameras. Photograph blurring is mainly because the objects photographed are located in the focal plane imaging system, we define it as defocus blur [1]. Traditional method of defocusing optical processing includes the gray-level histogram equalization and the compensation algorithm based on multiple linear regression model. Most of the methods recover images by convolution, however, polykeys exist during the deconvolution process [9], increasing the difficulty of image restoration. Wang Shoujue at el regard: Anything that you would get to know (including images, sounds, languages, status, etc.), if there exist two homologous but not the same objects, yet the differences between the two objects could be gradually varied or unquantized, (most things in macro world are like this, although just like the "apple output", we couldn't refine the production to one apple, yet when "apple output" is measured by weight, it could be considered as gradually varied), then between the two similar things, there must exist a gradual process, where all things belong to the same class. The law regarding homology continuity between the ontology are called as Principle of Homology-Continuity [7].

According [3] and [4], we can use the iteration method to restore the image. And In [5] and [6], they proposed take the image into some parts with the relationship of Relevancy of every point. The paper proposes a novel image restoration method based on homology continuity, which differs from the traditional method. This method takes images as a 
gather of point range, every point in the image and several defocusing blurring points are homologous, and they all meet the homology continuity principle. By the distribution relationship of the original image points and the derived points, we seek out a positive direction path of the former object's clear point, and the feasibility of this method is verified by repeated iteration. Theoretically, the more iterations we do, the more precise image it is, and time cost for image restoration are longer as compensation. However, noise are brought in during the process of degeneration, causing great impacts to the image continuity. That is to say, too much iteration times will enlarge noise objectively, thus the limitation of iteration times should also be considered.

\section{The Realization of Image Restoration Technology}

We assume two points in space $x_{1}\left(x_{1}, x_{2}, \ldots, x_{n}\right)$ (vector is represented as $\left.x_{1}\right)$, $Y_{1}\left(y_{1}, y_{2}, \ldots, y_{n}\right)$ (vector is represented as $\left.Y_{1}\right)$, as shown in the Figure 1, the linear vector equation passes the two points is:

$$
Y=Y_{1}+\lambda\left(Y_{1}-X_{1}\right),
$$

where $\lambda=\left|Y Y_{1}\right| /\left|Y_{1} X_{1}\right|$.

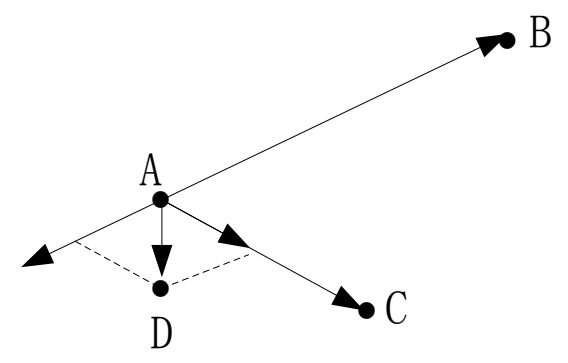

Figure 1. The Relationship of the Spatial Points' Location

If the three points in a known space are $A\left(x_{1}, x_{2}, \ldots, x_{n}\right), B\left(y_{1}, y_{2}, \ldots, y_{n}\right)$, $C\left(z_{1}, z_{2}, \cdots, z_{n}\right)$, then the path goes through could be expressed as:

$$
D=\alpha A+\beta B+\gamma C .
$$

Where $\alpha, \beta, \gamma$ are constant coefficient, generally $\alpha>0, \beta<0, \gamma>0$, and $\alpha+\beta+\gamma=1$ to ensure the image grey value remains the same. The equation could be

$$
D=A+k_{1}(B-A)+k_{2}(C-A),
$$

where $\alpha=1-k_{1}-k_{2}, \beta=k_{1}, \quad \gamma=k_{2}$.

The smaller value $k_{2}$ is, the bigger absolute value $k_{1}$ is, and the curve formed by $D, A, B, C$ is smoother. Thus, we could find the clearest point $D$ by adjusting the value of $k_{1}$ and $k_{2}$.

\section{Image Degradation Model}

In digital image processing, the principle of linear system in image processing is shown in Figure 2: 


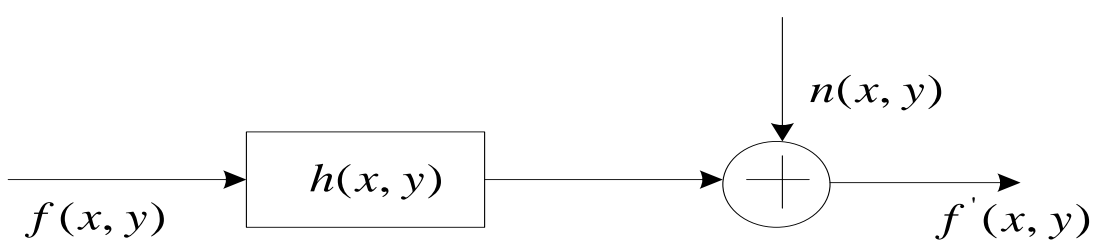

Figure 2. Image Degradation Model

Where $f(x, y)$ represents the original image, $h(x, y)$ is the image blur factor, $n(x, y)$ is the additional noise, $f^{\prime}(x, y)$ is the received image after transmission. Research shows that, image defocus blur caused by the optical system, the corresponding point spread function is a uniformly distribute circular spot. Generally, image blur is caused by defocusing, the image of a point source could be approximately looked as a disc[8], where the cylindrical point spread function is one of the most main, that is:

$$
h(x, y)=\left\{\begin{array}{c}
\frac{1}{\pi R^{2}}\left(x^{2}+y^{2} \leq R^{2}\right) \\
0\left(x^{2}+y^{2} \geq R^{2}\right)
\end{array}\right.
$$

In this paper, the above function is selected as the defocusing function.

\section{Image Restoration Principle Based on Homology Continuity}

Definition 1: If $f: x \rightarrow y$ is one-to-one related, in addition, $f$ and $f^{-1}$ are both continuous, then $f$ is called a homologous continuous path. As long as there exists a homologous path from $x$ to $y$, then we say that $x$ and $y$ are homologous, denoted by $x \cong y$.

Definition 2: The set of curves parallel to homologous continuous path is called homologous contiguous space, the mapping function of this space is denoted by $f:\left(x_{1}, x_{2}, x_{3}, \cdots, x_{n}\right) \rightarrow\left(y_{1}, y_{2}, y_{3}, \cdots, y_{n}\right)$.

Property 1: If plane $A$ passes $f:\left(x_{1}, x_{2}, x_{3}, \cdots, x_{n}\right) \rightarrow\left(y_{1}, y_{2}, y_{3}, \cdots, y_{n}\right)$, and receives plane $B$, then plane $A$ and plane $B$ possess homology continuity, denoted by $A \cong B$; If plane $A$ and plane $B$ possess homology continuity, then there at least exists a homologous contiguous space, which enables a random point on plane $A$ to map in plane $B$.

Definition 3: The collection of all images formed by image degradation models with the same image called homologous image space.

Property2: Suppose set $A=\left\{\left[\boldsymbol{a}(\boldsymbol{x})_{i, j}\right]_{M \times N} \mid \mathbf{1} \leq \boldsymbol{x} \leq \boldsymbol{n}\right\}$ and set $B=\left\{\left[b(y)_{i, j}\right]_{M \times N} \mid 1 \leq y \leq n\right\}$ are both the collection of $M \times N$ image gray level matrix, where $b(y)_{i, j}=h * a(x)_{i, j}, a(x)_{i, j} \in[1,255], a(x)_{i, j} \in A$, $b(y)_{i, j} \in[1,255], b(x)_{i, j} \in B, h$ is the point spread function, then $A \cong B$.

Definition 4: Suppose in an image, we change point $a$ in plane $A$ to point $b$ according to the degradation model, then $a$ is called the clear point of $b, b$ is the fuzzy point of $a$, denoted by $a \stackrel{M}{\longrightarrow} \rightarrow b, b \stackrel{x}{\longrightarrow} \rightarrow a$. The image $B$ constituted by $b$ is the 
blurred image of image $A$, denoted by $A \stackrel{M}{\longrightarrow} B$. Conversely, image $A$ is the clear image of image $B$, denoted by $B \stackrel{x}{\longrightarrow} \rightarrow A$.

Definition 5: Let $\mathrm{A}$ and $\mathrm{B}$ be the matrix of two images in a same homologous image space. If for every number $\varepsilon>0$, there is $|B-A|<\varepsilon$. Then we say that, image $A$ and image $B$ are equivalent, denoted by $A=B$.

\section{Image Restoration Algorithm Based on Homology Continuity}

\subsection{Algorithm Framework}

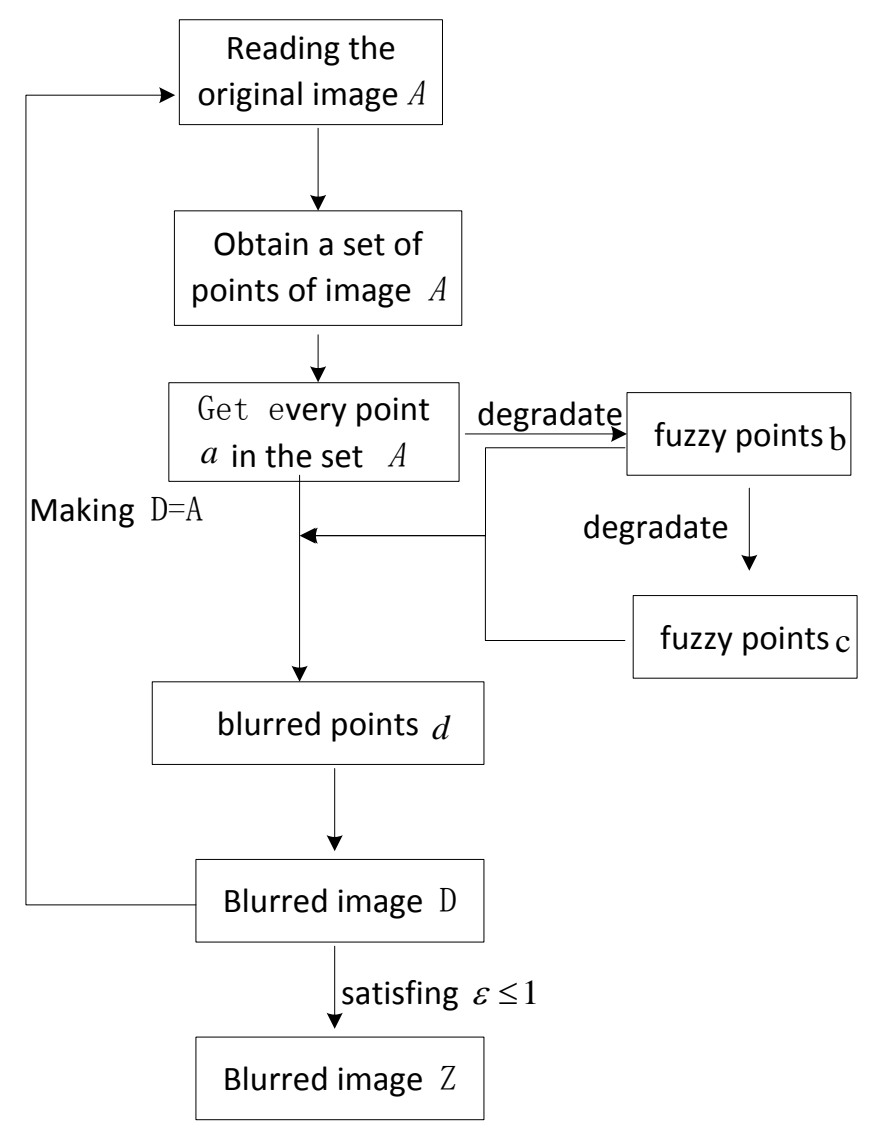

Figure 3. Algorithm Framework

\subsection{The Concrete Process of the Algorithm}

Step1: Reading the original image $A$ as a set of points in $A=\left\{\left[a(x)_{i, j}\right]_{M \times N}\right\}$.

Step2: Using the quality model to obtain fuzzy points $\mathrm{b}(y)_{i, j}$ and $\mathrm{c}(z)_{i, j}$ of every point $a(x)_{i, j}$ in the set $A$. Points $a, \mathrm{~b}, \quad \mathrm{c}$ should satisfy $a \stackrel{M}{\longrightarrow} \rightarrow b \stackrel{M}{\longrightarrow} \rightarrow c$.

Step3: Selecting appropriate parameters $\alpha, \beta, \gamma$, then calculate $d=\alpha a+\beta b+\gamma c$, so as to obtain the first restored point $d$, then $a \stackrel{x}{\rightarrow} \rightarrow d$.

Step4: Using the set $D=\left\{\left[\mathrm{d}_{i, j}\right]_{M \times N} \mid 1 \leq x \leq n\right\}$ comprised by the whole points $d$ to restore image $D$, and observe its effect.

Step5: Making $D=A$, then back to Step2 repeatedly. 
Step6: Running these above-mentioned steps until the restored image has satisfied definition six, then stop iteration, outcome image $D$.

According to the mentioned algorithm, the image obtained from every iteration would be clearer then the last one. With appropriate times of iterations, the effect will be much closer to the original image.

\section{Image Quality Evaluation Method}

The meaning of the image quality mainly include two aspects [2]: The fidelity of the image and the intelligibility of the image. To make reasonable assessment of various links in image processing, the research of image quality assessment has become one of the most fundamental technologies in image information engineering. In the image restoration technology, we hope the restored image to resemble the original image, so we use the objective criteria to evaluate the image quality and recovery algorithm. The objective evaluation of image quality is to build the mathematical model according to the human eye subjective visual system, and compute the image quality according to the specific formula. The traditional objective image quality evaluation method mainly includes MSE (mean squared error) and PSNR (peak signal to noise rate). The paper adopts the MSE method to evaluate the quality of the restored image. The mean square error method firstly calculates the mean square value of the original image and the distorted pixels' difference value, then ascertain the distortion degree of the distorted images by the mean square value and the peak signal to noise rate. The computation formula is as following:

$$
M S E=\frac{1}{M \times N} \sum_{0 \leq i \leq N} \sum_{0 \leq j \leq M}\left(f_{i j}-f_{i j}^{\prime}\right)^{2}
$$

Where $M 、 N$ represents the width and length of the image, $f_{i j}$ is the pixel value of the original image, $f_{i j}^{\prime}$ is the pixel value of the quality reduced image.

\section{Experiment and Analysis}

The paper uses Python 2.7, combing PIL image processing library [10] to realize the above algorithm, and utilized the first blurred image generated by the original image as the experimental read blurred image $A$.

The specific effect is shown in Figure 4. In the images, (1) is the original image; (2) is the experimental read blurred image $A$. After 10 cycles the iteration terminates. (3) (12) is the output restored image in the iteration process. Figure 5 is the value relationship between the curve and the number of iterations between the image and the original image iterative reduction. By the curve can be seen, along with the increase of the number of iterations, the value of MSE become smaller.

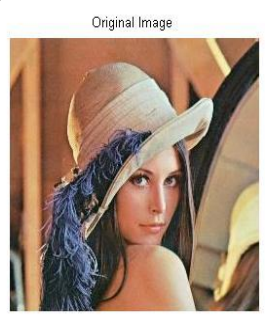

(1) The original image

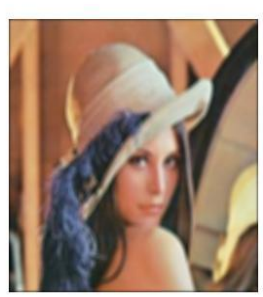

(2) Read in image A

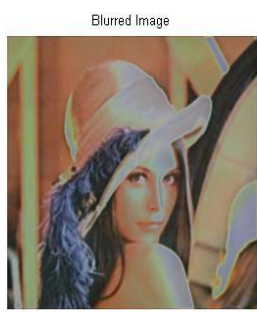

(3) 


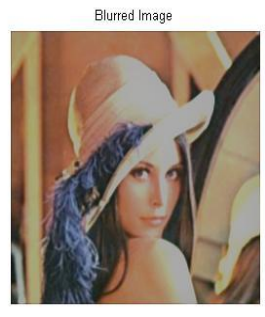

(4)

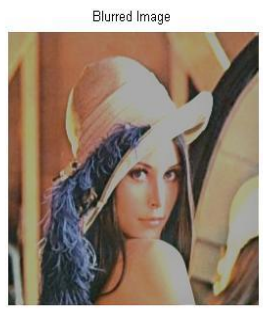

(7)

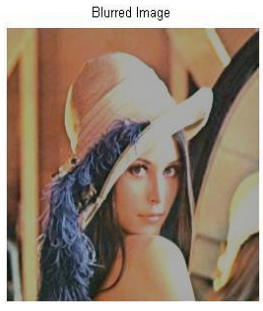

(10)

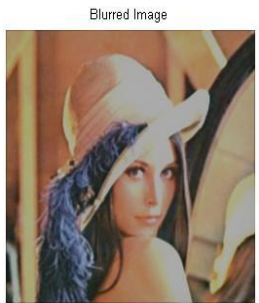

(5)

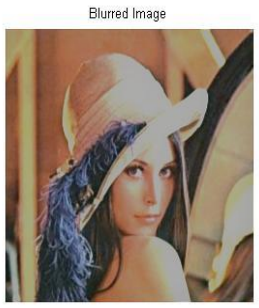

(8)

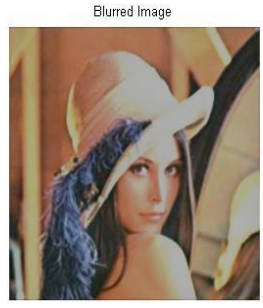

(11)

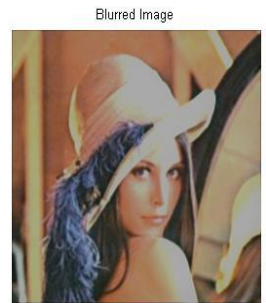

(6)

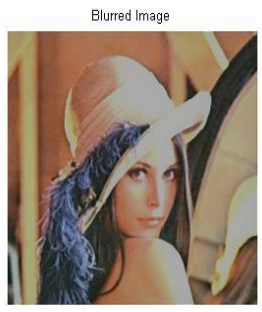

(9)

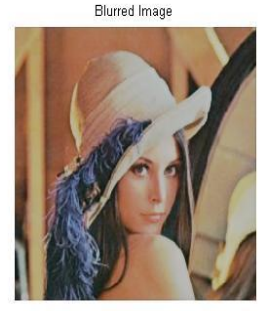

(12)

Figure 4. Image Restoration Effect Picture

Experiments show that in the process of iteration, the images disposed by iteration are much closer to the original image effect, while the actual visual effect is also proved this point.

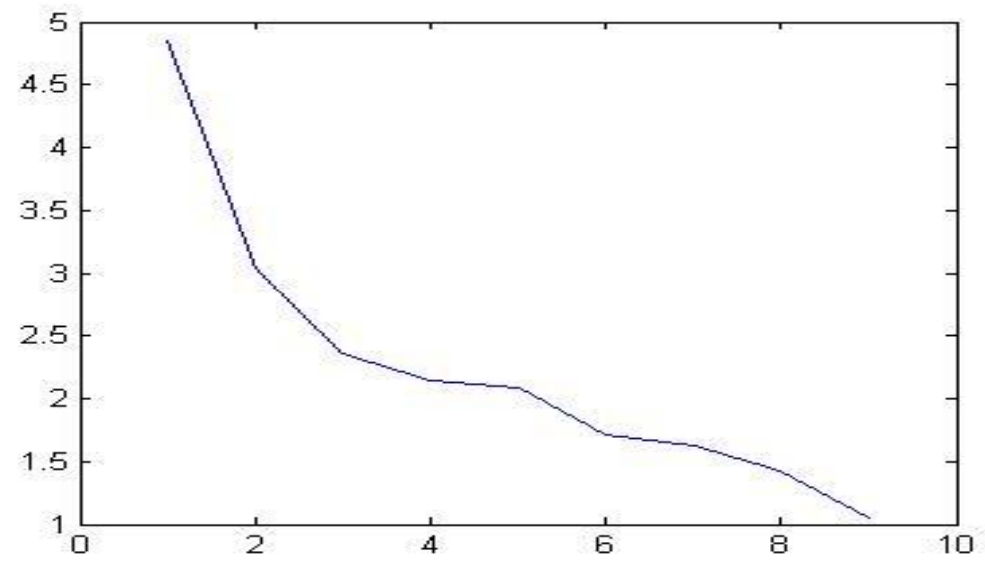

Figure 4. Relationship between the Number of Iterations and the MSE 
It is proved in practice that the homologous continuous path consisting of each point in a image and the points formed by the point through degradation model can retrieve the starting point of the path. Thus the image space formed by the points on the homologous continuous path is also satisfy this property. So the image restoration method is feasible.

\section{Conclusion}

Since in the actual use process, the traditional recovery image method needs to know the noise power and original image's power in advance to restore image better. Since the two values are both unknown or unpredictable, thus this approach is hard to realize [3]. Compared with deconvolution methods, this method avoids the problem of multiple solutions [8]. The paper proposes an image restoration method based on homology continuity, as long as the image obscure reasons meet Definition 1, thus the suggested method possesses universality. The image quality handled by the experimental data is pleasing, which provides a novel method of restore blurred images in digital image library. Because the number of subjects in the database of digital picture is very big, it is difficult for us to estimate the related parameters of according to each picture, this method is more general. But it needs further study in the uniqueness of image restoration with the equivalent conditions of the image.

\section{Acknowledgments}

This paper is supported by the research grant (No.14DZ2261200) from Shanghai Government and Shanghai Institute of Materials Genome, Foundation of Science and Technology Commission of Shanghai Municipality under Grant No. 14590500500, Natural Science Foundation of Shanghai under Grant No. 15ZR1415200, and Young University Teachers Training Plan of Shanghai Municipality under Grant No. ZZSD13008.

\section{References}

[1] Banham M. and Katsaggelos A., "Digital Image Restoration. IEEE Signal Processing Magazine", vol. 14, no. 2, (1997).

[2] A. M. Eskicioglu and P. S. Fisher, "Image Quality Measures and Their Performance", IEEE Transactions on Communication, (2000).

[3] C. Yunlong, W. Ping and W. Peng, "PMAP based on the nonlinear iteration of L - R quality image restoration algorithm", Computer Engineering, vol. 36, no. 4, (2010), pp. 2.

[4] Wang S. J., Cao Y. and Huang Y., "A novel Image Restoration Approach Based on Point Location in High-dimensional Space Geometry", The Second Int. Conf on Neural Networks and Brain[C], (2005).

[5] Z. Lin, Z. Yueting, W. Jiangqin, Y. Zhenchao and W. Fei, "An image retrieval method based on sparse typical correlation analysis”, Journal Software, vol. 23, no. 5, (2012), pp. 1295-1304.

[6] Z. Shaomin, Z. Dazhe, Z. Hong and L. Shukuan, "Mutual information similarity combination image retrieval algorithm inspired", Chinese journal of image and graphics, vol. 16, no. 10, (2011), pp. 857863.

[7] W. Shoujue, "First step to Multi-Dimensional Space Biomimetic Informatics", Beijing, National Defense Industry Press.

[8] R. C. Gonzalez and R. E. Woods, "Digital Image Processing. Second Edition", Beijing: Publishing House of Electronics, (2002).

[9] W. Qiao, "Digital Image processing”, Beijing: Science press, (2009), pp. 133-155.

[10] http://www.pythonware.com/products/pil/. effbot.org. Python Imaging Library 1.1.7. 


\section{Authors}

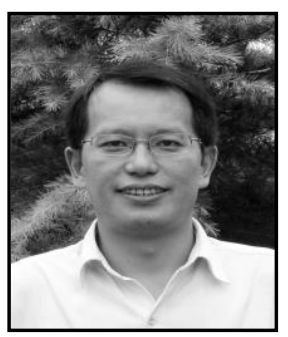

Dr. Yonghua Zhu, vice Professor. He received the B.S. at Xi' an Jiaotong University in 1990, the M.S. at Tongji University in 1993 and Ph.D. at Shanghai University. He is working in the School of Computer Engineering and Science of Shanghai University. His current research interests are focusing on high performance computing, network computing, interconnected network design, Communication and Information Engineering, Intelligent Controlling. Over the past ten years, he has published over 20 technical papers in the related fields. He will keep on the research work addressing Cross-disciplinary of communication, computer and automation.

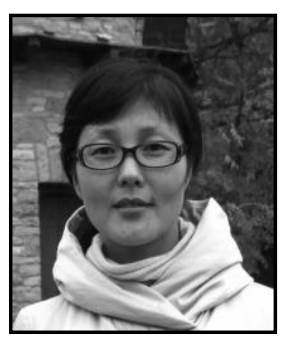

Dr. Pin Wu, vice Professor. She received the B.S. and Ph.D. at Nanjing University of Science and Technology in 1998 and 2003. She had worked in Zhejiang University as a post doctor for two years, and had worked in Michigan State University as a senior visiting scholar for one year. She is working in the School of Computer Engineering and Science of Shanghai University now. Her current research interests are focusing on image processing, high performance computing (HPC), computational fluid dynamics (CFD) and so on. Over the past ten years, she has published over 30 technical papers in the related fields. She will keep on the research work addressing Cross-disciplinary of computer and mechanics.

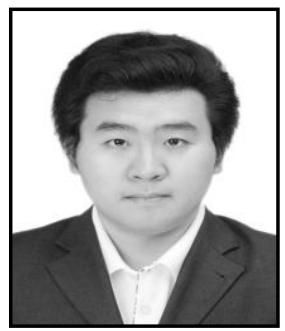

Dr. Honghao Gao, received the $\mathrm{PhD}$ degree in the School of Computer Engineering and Science of Shanghai University, Shanghai, China, in 2012. His research interests include Web service and model checking. 